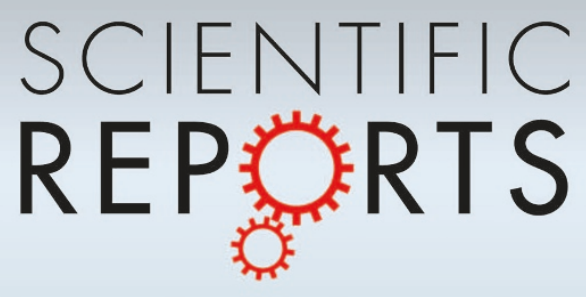

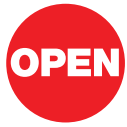

SUBJECT AREAS:

MECHANICAL AND

STRUCTURAL PROPERTIES

AND DEVICES

SURFACE CHEMISTRY

MECHANICAL ENGINEERING

APPLIED PHYSICS

Received

22 January 2013

Accepted

3 April 2013

Published

25 April 2013

Correspondence and requests for materials should be addressed to

Z.H.Y. (yaozh@

tsinghua.edu.cn)

\section{Control of Superhydrophilic and Superhydrophobic Graphene Interface}

\author{
Jing Dong' ${ }^{1}$ Zhaohui Yao' , Tianzhong Yang ${ }^{2}$, Lili Jiang ${ }^{2} \&$ Chengmin Shen ${ }^{2}$
}

\begin{abstract}
'School of Aerospace, Tsinghua University, Beijing 100084, PR China, ${ }^{2}$ Beijing National Laboratory of Condensed Matter Physics, Institute of Physics, Chinese Academy of Sciences, Beijing 100190, PR China.
\end{abstract}

Superhydrophobic and superhydrophilic properties of chemically-modified graphene have been achieved in larger-area vertically aligned few-layer graphene nanosheets (FLGs), prepared on Si (111) substrate by microwave plasma chemical vapor deposition (MPCVD). Furthermore, in order to enhance wettability, silicon wafers with microstructures were fabricated, on which graphene nanosheets were grown and modified by a chemical method to form hydrophilic and hydrophobic structures. A superhydrophilic graphene surface (contact angle $0^{\circ}$ ) and a superhydrophobic graphene surface (contact angle $152.0^{\circ}$ ) were obtained. The results indicate that the microstructured silicon enhances the hydrophilic and hydrophobic wettabilities significantly.

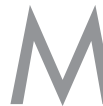

any plants' leaves have a special function of self-cleaning, especially the lotus, as has been noted for thousands of years. We call this kind of phenomenon the 'Lotus effect.' A leaf surface that has the 'Lotus effect' is superhydrophobic ${ }^{1}$, which indicates that the contact angle (CA) with a water drop is larger than $150^{\circ}$. One important feature of such a surface is anti-contamination, which means that contamination can be easily washed away by liquid. Barthlot and Neihuis ${ }^{2,3}$ studied the microstructure of lotus leaves and concluded that a lotus leaf s mastoid structure in micrometer scale is the main reason for the lotus effect. Superhydrophobic and superhydrophilic surfaces are very useful in many applications, such as waterproof surfaces, anti-contamination surfaces, coatings and biomedical devices.

Graphene is a two-dimensional crystal, which has been regarded as unstable ${ }^{4-6}$. In 2004, the discovery of graphene demonstrated that a single sheet of carbon atoms is stable at room temperature ${ }^{7,8}$. Graphene has attracted great interest because it has many extreme properties, such as mechanical stiffness ${ }^{9}$, high operating bandwidth ${ }^{10}$, and high thermal ${ }^{11}$ and electrical ${ }^{12}$ conductivity. In the past decade, different qualities of graphene were prepared through various routes, including mechanical exfoliation ${ }^{6}$, epitaxial growth on silicon carbide ${ }^{13}$, epitaxial growth on single crystal metal substrates ${ }^{14}$, chemical vapor deposition (CVD $)^{15}$ and reduction of graphite oxide $(\mathrm{GO})^{16}$. Pristine graphene is similar to a natural lotus leaf in that it exhibits hydrophobicity. But its wetting ability can be changed and enhanced by different modifications. First, multiple synthesis methods were used to get graphene with different microstructures, which enhanced the hydrophobic/hydrophilic properties; second, chemical modifications were presented to further improve the hydrophobic/hydrophilic properties. In previous studies, different wetting characteristics of graphene were obtained through chemical modification of Graphite Oxide (GO), aerogels of graphene and graphene-resin composite. GO has many hydrophilic functional groups such as carboxyl and hydroxyl, and further chemical modification can change these functional groups and get different interface properties. Moreover, GO has a flake-like structure in micron scale, which can be controlled to enhance the graphene's particular wetting ability. Rafiee ${ }^{17}$ used a rapid thermal expansion route to expand and reduce the GO to prepare few-layer graphene. Then acetone or molecular water was attached to the surface of graphene sheets to change their wettability from superhydrophobic to superhydrophilic. Wong's group reported that GO sheets were modified using octadecylamine (ODA) to form a superhydrophobic graphene surface ${ }^{18}$. Zhao et al., synthesized a superhydrophobic composite which has special structure in micron scale combining vinylidene fluoridehexafluoropropylene (PVDF-HFP) and graphene ${ }^{19}$. This material has a CA of $152^{\circ}$. Jin's group ${ }^{20}$ also got a porous composite combining graphene and PVDF which is superhydrophobic. Liu's group ${ }^{21}$ got a composite combining graphene and 2-methoxy-5-(20-ethyl-hexyloxy)-1,4-phenylene vinylene(MEH-PPV) particle, which has a CA of $152^{\circ}$. Graphene aerogel has a great specific surface area, which can enhance its wetting a lot. $\operatorname{Lin}^{22}$ got superhydrophobic graphene aerogel by rapid thermal expansion of GO, and then modified it by $1 \mathrm{H}, 1 \mathrm{H}-2 \mathrm{H}, 2 \mathrm{H}$ perfluorodecyl-trichlorosilane. The CA of graphene aerogels can be larger than $160^{\circ}$. 
Although some research about superhydrophilic and superhydrophobic graphene was presented in recent years, there is little research about the wetting property of graphene synthesized by the MPCVD method. MPCVD is commonly used in industry, since it can prepare wafer-size homogeneous samples. MPCVD is used to synthesize fewlayer vertical graphene nanosheets on different kinds of bases, such as silicon ${ }^{23}$, nickel and titanium ${ }^{24}$. Maslesevic ${ }^{23}$ has synthesized vertical few-layer graphene by the MPCVD method, and his experiment showed that the graphene synthesized by MPCVD has a great field emission property. The microstructure of MPCVD graphene has vertical nanosheets, sharp edges and a large surface area, which may significantly improve the surface's wetting ability. But no one has reported the wetting property of MPCVD graphene. Moreover, there is little research about the wettability of graphene on a patterned surface with microstructure. It has been reported that a silicon surface with micropillars has much better hydrophobicity than a smooth silicon surface ${ }^{25}$. Graphene synthesized on a silicon microstructure may have better wettability than graphene synthesized on a smooth silicon surface.

Although MPCVD is a well developed technique and has been used by other groups, the recent investigations of vertical graphene prepared by MPCVD mainly focused on its field emission properties. The superhydrophilic and superhydrophobic properties of vertical graphene on the silicon surface have not been reported. In this paper, we report the control the superhydrophilic and superhydrophobic property of graphene on a wafer-size silicon surface. This may expand the application potential of graphene. Vertically aligned few-layer graphene nanosheets (FLGs) were prepared by microwave plasma chemical vapor deposition on a patterned silicon substrate. Then the surface of the FLGs was modified using a chemical method to get better superhydrophilic and superhydrophobic performance. The graphene's wetting characteristic can be tuned from superhydrophilic to superhydrophobic. In order to compare the influence of the substrate on the wetting properties of graphene, we used silicon with micron-scale structures as the substrate to fabricate FLGs.
Growing FLGs on the microstructured substrate can enhance the special wetting properties.

\section{Results}

The vertical few-layer graphene nanosheets were successfully prepared on the Si substrate using MPCVD. Figure 1(a) shows SEM images of FLGs grown on the $\mathrm{Si}(111)$ in which we can clearly see that the surface of the FLGs is smooth and uniform. The size of the graphene sheet is about $1 \mu \mathrm{m}$ and a few layers thick. A cross sectional SEM image of FLGs is shown in Fig. 1(c). It can be found that the sheets grew vertically on the substrate and the height of each sheet is about $1 \mu \mathrm{m}$.

Raman spectrum was used to confirm the thickness of FLGs. The Raman spectrum of FLGs is shown in Fig. 1(b). Three peaks are observed: $\mathrm{G}$ peak at $1583 \mathrm{~cm}^{-1}$, D peak at $1348 \mathrm{~cm}^{-1}$ and $2 \mathrm{D}$ peak at $2699 \mathrm{~cm}^{-1}$. The intensity of the $\mathrm{D}$ peak relates to disordered carbon content and defects in the graphene. The $G$ peak at $1583 \mathrm{~cm}^{-1}$ represents the graphite content. The ratio of the intensities of the $D$ and $G$ peaks $\left(\mathrm{I}_{\mathrm{D}} / \mathrm{I}_{\mathrm{G}}\right)$ shows the number of disorders and defects in the graphene. The intensity of the $2 \mathrm{D}$ peak relates to the layer thickness of the graphene sheets. The ratio of the intensities of the $2 \mathrm{D}$ and $\mathrm{G}$ peaks $\left(\mathrm{I}_{2 \mathrm{D}} / \mathrm{I}_{\mathrm{G}}\right)$ indicates the quality and number of layers of the sheets $^{26-30}$. From the Raman spectrum analysis result, we find that the $2 \mathrm{D}$ band ratio $\mathrm{I}_{2 \mathrm{D}} / \mathrm{I}_{\mathrm{G}}$ is about 1.0. Therefore, an as-prepared vertical graphene sheet has about 10 layers $^{29,30}$. High resolution transmission electron microscopy (HRTEM) was also used to present the real thickness of the as-prepared graphene nanosheet directly. The HRTEM image in Fig. 1(d) shows that the thickness of one typical graphene nanosheet is 9 layers, which confirms that the Raman spectrum gives us valid evidence of the thickness of the as-prepared FLGs.

The vertical graphene sheet film was modified through a chemical method. The detailed modification process is described in Fig. 2. The first step is an oxidation process, in which FLGs are oxidized in a solution of sulfuric acid, potassium permanganate and sodium
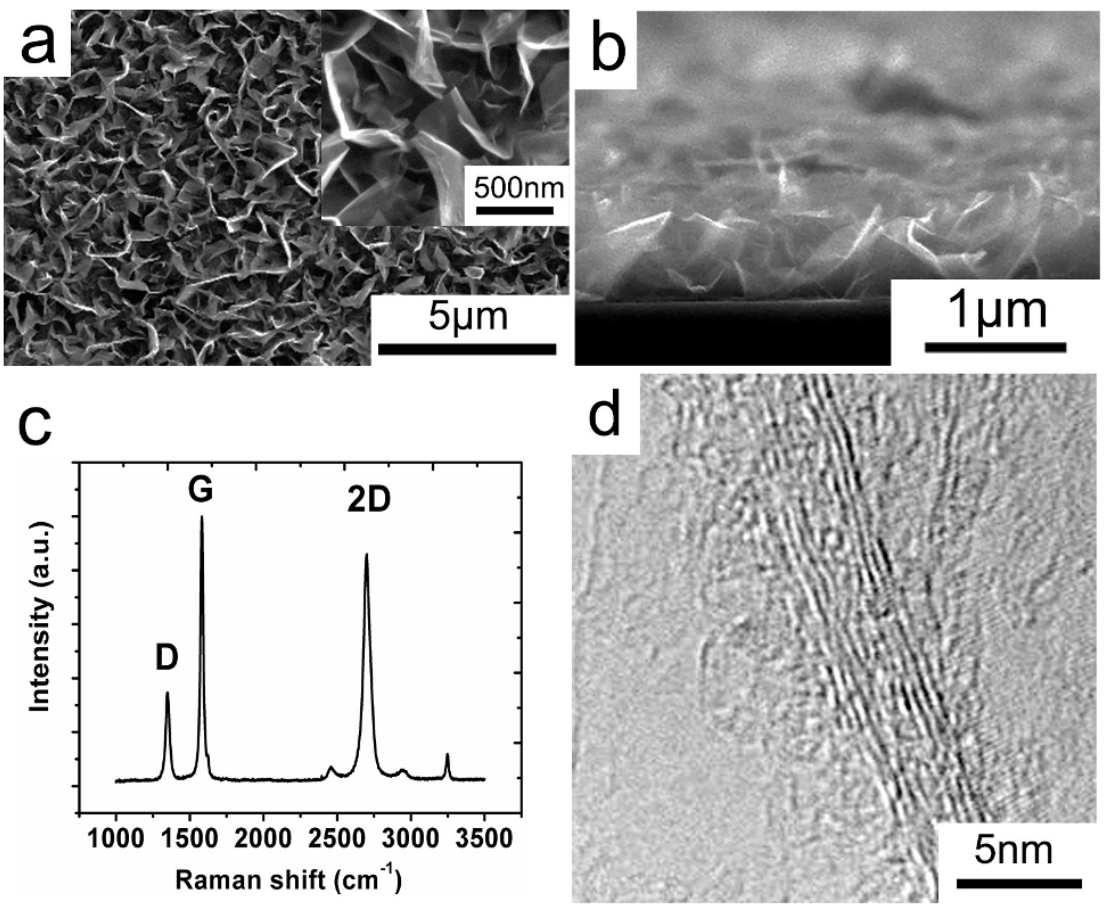

Figure 1 SEM images of FLGs and Raman spectrum of FLGs. (a) SEM image of FLGs on smooth silicon surface. The size of graphene nanosheet is about $500 \mathrm{~nm}$ to $1 \mu \mathrm{m}$. (b) SEM image of cross section of FLGs on substrate. The height of FLGs is about $1 \mu \mathrm{m}$. (c) Raman spectrum of FLGs, G peak at $1583 \mathrm{~cm}^{-1}$, D peak at $1348 \mathrm{~cm}^{-1}$ and 2D peak at $2699 \mathrm{~cm}^{-1}$. (d) HRTEM image of a typical graphene nanosheet of FLGs. It can be seen clearly that the edges terminated with nine-layers graphene. 


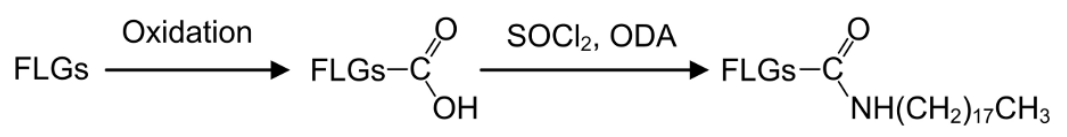

Figure 2 Schematic diagram of the chemical modification of FLGs.

nitrate. We call them Oxide-FLGs (O-FLGs) after they are oxidized. The second step is to acyl-chloridize the O-FLGs in a solution of thionyl chloride and DMF, then in melted ODA. These products are called the ODA-modified FLGs (ODA-FLGs).

SEM images of chemically modified FLGs are shown in Fig. 3(a-b). We can clearly observe that the surface and structure of the FLGs has not been damaged by the chemical modification. The sheet-like structure of FLGs is preserved after chemical reaction. The micro sheet structure is preserved while functional groups on FLG have been greatly changed after oxidation and ODA modification. On the other hand, the molecular structure of the FLGs has been significantly changed by the reaction, especially in the first step of oxidation. Xray diffraction technology was used to characterize the crystal structure of FLGs. Figure 3(c) presents the XRD patterns of FLGs and OFLGs. A diffraction peak at $26.4^{\circ}$ can be observed, corresponding to the (002) plane of natural graphite. In the XRD result of O-FLGs, the diffraction peak at $26.4^{\circ}$ has disappeared, indicating that the typical layer structure of graphite does not exist. The interpretation of this phenomenon is that oxidation inserts oxygen-containing functional groups into the graphene layers. The distances of graphene layers have been changed separately and are different. So there is no sharp peak in the XRD pattern.

Raman spectra of modified FLGs show that the quality of FLGs has been changed. In Fig. 3(d) we can see that the 2D peak significantly decreases and the $\mathrm{D}$ peak gradually increases. The decrease of the 2D peak indicates that many functional groups have formed on the surface of the O-FLGs. The intensity of the D peak is clearly increased, which demonstrates an abundance of new defects, edges and disorders in the graphene nanosheets, since oxidation breaks up the sp 2 carbon network, and many sp3 carbon atoms have been added into the FLGs. Some other peaks which are not obvious indicate that some other atoms and bonds exist, due to the presence of oxygen-containing functional groups.

To enhance the hydrophobic and hydrophilic abilities of FLGs, silicon microstructures were designed and fabricated. Then FLGs were grown on the microstructures. The SEM images in Fig. 4(a(b) show that FLGs grew homogeneously on the silicon substrate. Then these FLGs were modified similarly by the steps above. The oxidizing solution in the first step cannot dissolve silicon, and the molten ODA which is alkaline cannot corrode (111) silicon since there is no (100) surface, which is the only surface alkaline solution can dissolve. So the silicon microstructure is preserved after the reaction.

Since the functional groups on FLGs are changed in different stages of the reaction, the wettabilities of different kinds of FLGs among our samples differ significantly. The CA of unmodified FLGs, O-FLGs and ODA-FLGs have been measured. The CA of the unmodified FLGs is $132.9^{\circ}$, showing that they are hydrophobic. This result is easy
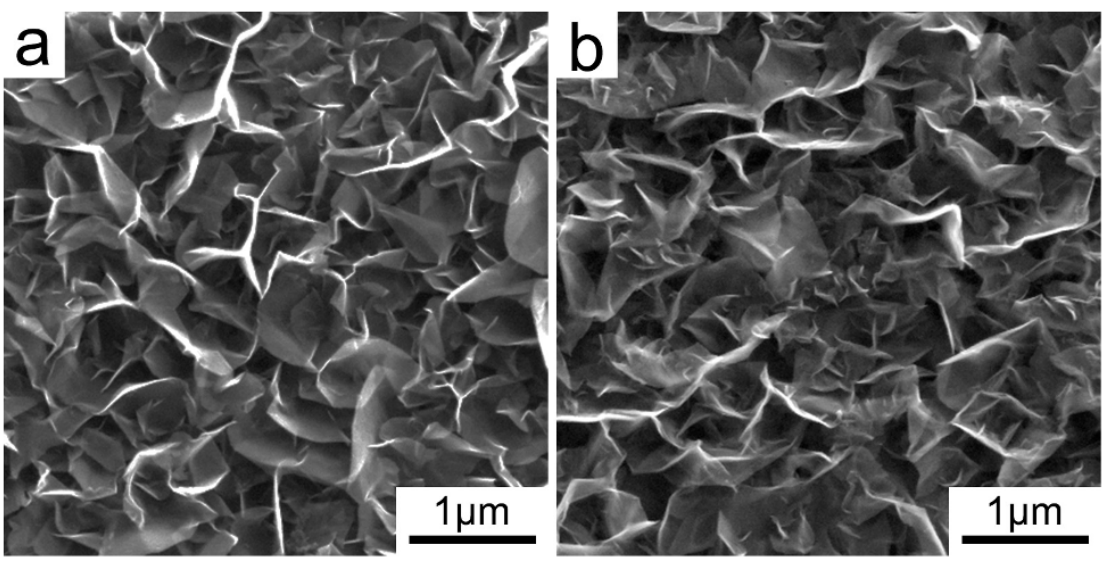

C

d
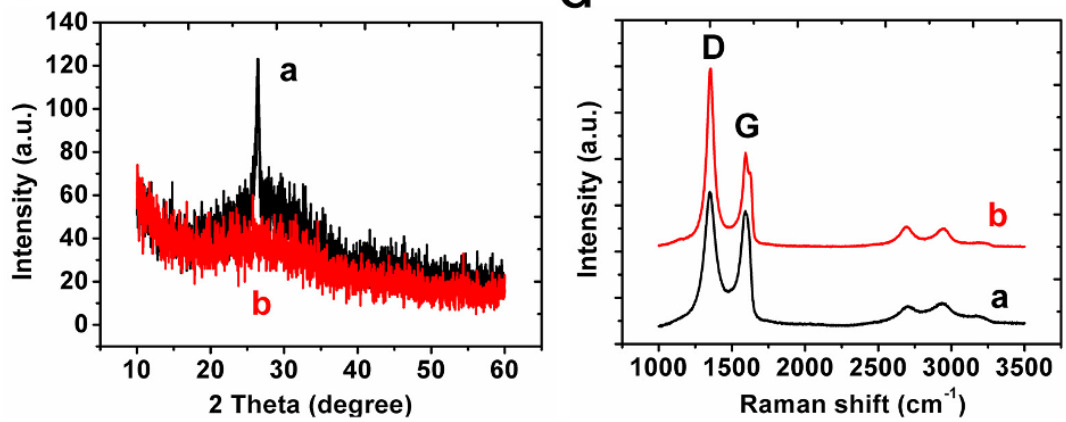

Figure 3 SEM image of O-FLGs and ODA-FLGs, and XRD and Raman spectrum of O-FLGs and ODA-FLGs. (a) SEM image of O-FLGs on substrate. The structure of O-FLGs is similar to FLGs. (b) SEM image of ODA-FLGs on silicon substrate. The SEM image shows that the oxidation of reaction with ODA does not damage the few-layer structure of FLGs. (c) XRD result of FLGs and O-FLGs. (a) is FLGs, a peak at $26.4^{\circ}$ can be found. (b) is O-FLGs and there is no significant peak at $26.4^{\circ}$. (d) Raman spectrum of O-FLGs and ODA-FLGs. (a) is O-FLGs, and (b) is ODA-FLGs. 2D peak disappears and ID/IG is raised significantly. 


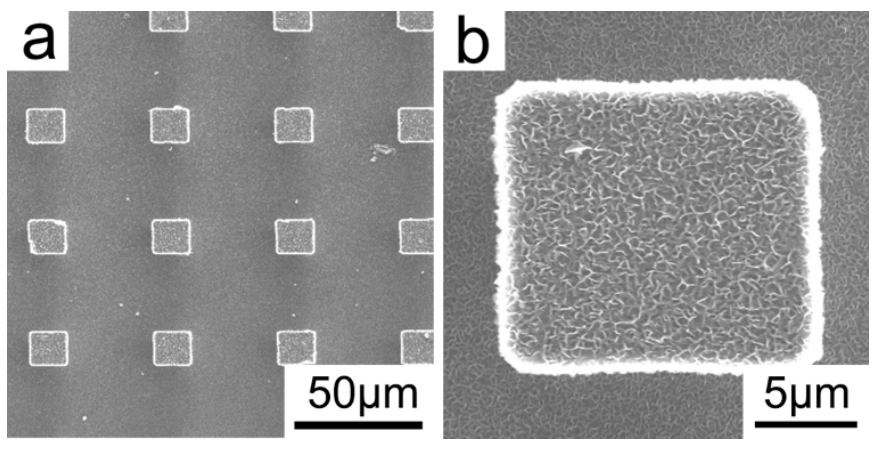

Figure 4 | SEM images of silicon microstructure. (a)-(b) SEM images of FLGs on silicon microstructures. FLGs grew uniformly on silicon structure including the sides and bottom of the silicon pillars.

to understand, since pure graphene without any oxygen-containing functional groups is hydrophobic, and the nanosheet-like structure enhances the hydrophobicity. Figures 5(a)-(c) show droplets on smooth silicon surfaces that have different kinds of FLGs on them. The CA of O-FLGs is $53.1^{\circ}$, since many functional groups such as carboxyl and hydroxyl, which are hydrophilic, have been added onto the FLGs. Carboxyl and hydroxyl are hydrophilic because the oxygen significantly raises the surface energy. On the other hand, the CA of ODA-FLGs is $133.7^{\circ}$, indicating that ODA-FLGs are hydrophobic. A surface that has alkane chains is hydrophobic since the hydrogen reduces the surface energy. The large CA shows that alkane chains lower the surface energy. The CA of ODA-FLGs is significantly larger than that of O-FLGs but is similar to the CA of unmodified FLGs. This result indicates that the hydrophobic property of FLGs is not improved a lot by the reaction - they are similar in wettability. Although FLGs and ODA-FLGs are similar in CA and wettability, their differing Raman spectra have already shown that they are different substances. Considering that ODA molecules should make the hydrophobicity of ODA-FLGs better than that of simple FLGs, the similar CA of FLGs and ODA-FLGs should be attributed to the oxygen functional groups remaining on ODA-FLGs.

The CAs of FLGs, O-FLGs and ODA-FLGs on microstructures were also measured. Figures 5(d)-(f) show droplets on silicon microstructures which have FLGs. The CA of FLGs on microstructures is $149.8^{\circ}$. Comparing this to the $132.9^{\circ} \mathrm{CA}$ of FLGs on smooth silicon substrate, we can see that the microstructure enhances the hydrophobicity. The CA of O-FLGs on microstructure is $0^{\circ}$, meaning this
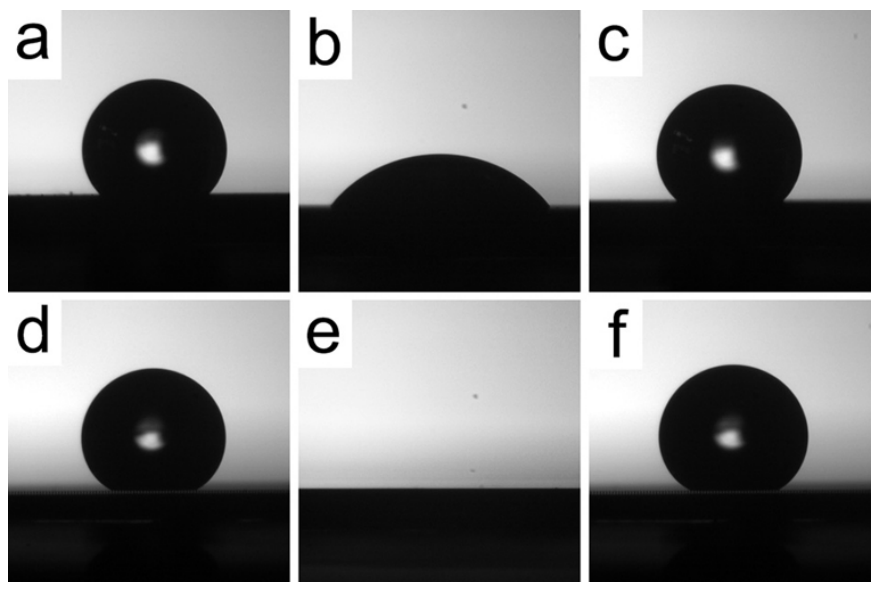

Figure $5 \mid$ CA of FLGs, O-FLGs and ODA-FLGs. (a) FLGs on smooth silicon substrate, $\mathrm{CA}=132.9^{\circ}$. (b) O-FLGs on smooth silicon substrate, $\mathrm{CA}=53.1^{\circ}$. (c) ODA-FLGs on smooth silicon substrate, $\mathrm{CA}=133.7^{\circ}$. (d) FLGs on microstructure, $\mathrm{CA}=149.8^{\circ}$. (e) O-FLGs on microstructure, $\mathrm{CA}=0$. (f) ODA-FLGs on microstructure, $\mathrm{CA}=152.0^{\circ}$. surface is superhydrophilic and it can be totally wetted by water droplets. The surface is more hydrophilic because of the microstructure. The CA of ODA-FLGs on the microstructure is $152.0^{\circ}$, meaning this surface is superhydrophobic. The microstructure also enhances the hydrophobicity of ODA-FLGs. The CA results prove that the hydrophobicity of the graphene has been improved a little by the ODA modified surface (from $149.8^{\circ}$ to $152.0^{\circ}$ on microstructure). The reason, as explained before, is that there are still some oxygen functional groups on ODA-FLGs, which reduce the CA.

\section{Discussion}

Wenzel $^{31}$ and Cassie ${ }^{32}$ have each offered a theory of the wetting of a surface which has structure. Wenzel's model describes the wettability of the surface which has been totally wet by liquid, and there are no gas bubbles between the liquid and the solid surface. On the other hand, Cassie's model describes the wetting of a surface in which gas bubbles reside between the liquid and the solid, and the surface is not totally wet. Figure 6(a) shows schematics of the Wenzel and Cassie contact models. In Wenzel's model, the CA of a smooth surface and the CA of a surface with structure satisfy

$$
\cos \theta_{w}=r \cos \theta
$$

where $\theta_{w}$ is the CA of the surface with structure, $\theta$ is the CA of the smooth surface, and $\mathrm{r}$ is the roughness ratio, which is defined by the ratio of the true surface area of the structure to its projection area. For surface which has microstructure and $r>1$, the structure makes the CA bigger if $\theta>90^{\circ}$, and makes the CA smaller if $\theta<90^{\circ}$. In Cassie's model, the CA of a smooth surface and the CA of a surface with structure satisfy

$$
\cos \theta_{c}=f_{1} \cos \theta-f_{2}
$$

where $\theta_{c}$ is CA of surface with structure and $\theta$ is CA of smooth surface, $f_{1}$ is ratio of the area of the solid-liquid interface to the total projection area, $f_{2}$ is the ratio of the area of the solid-gas interface to the total projection area, and $f_{1}+f_{2}=1$. The Cassie model tells us the CA will increase if there is gas between the solid and the liquid. According to the principle of minimum energy, the real contact mode will be either Cassie or Wenzel, depending on which one's

\section{a}

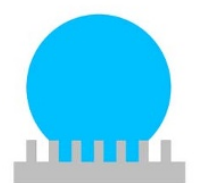

Wenzel
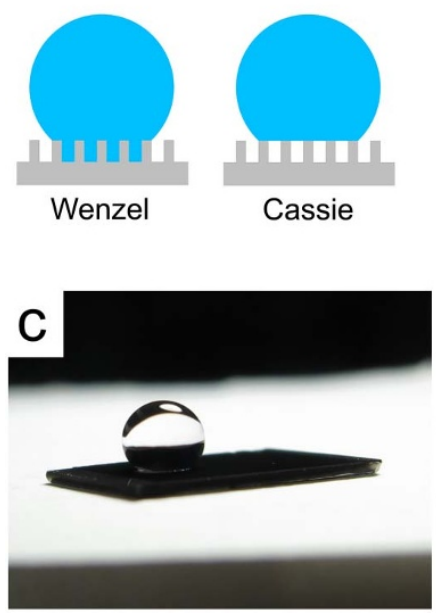

Cassie b

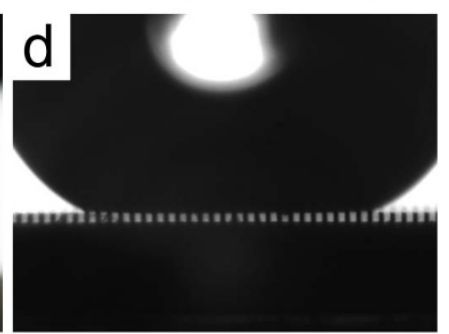

Figure 6 Schematics of several contact modes and the true contact mode. (a) Schematics of Wenzel and Cassie contact models. (b) Schematic of the silicon microstructure. (c) Photo of droplet on ODA-FLGs grown on silicon microstructure. (d) Optical image of the contact line of ODA-FLGs on microstructure. Gap with gas can be found between droplet and solid surface. 
energy is lower. An optical microscope can also be used to determine the contact mode.

For O-FLGs on silicon microstructure, since O-FLGs are hydrophilic, the surface is easily wetted, and the contact mode is Wenzel mode. According to the shape and size of our structure, the contact angle can be predicted by

$$
\cos \theta=A(1+\cos \theta)-1 .
$$

where $A$ is a parameter determined by this structure:

$$
A=\frac{1}{\left[\left(\frac{b}{a}\right)+1\right]^{2}}, a=15 \mu m, b=35 \mu m
$$

Figure 6(b) shows a schematic of the silicon microstructure. The CA of O-FLGs on a smooth surface is $53.1^{\circ}$, so the predicted the CA of $\mathrm{O}$-FLGs on microstructure is $0^{\circ}$. The experiment gives the same result. For ODA-FLGs on microstructures, the contact mode is determined by the surface energy. Optical microscope image Fig. 6(d) shows that there is air between the droplet and the silicon structure, so we assume the contact mode of ODA-FLGs on microstructures is the ideal Cassie mode. According to the shape and size of our structure, the contact angle can be predicted by

$$
\cos \theta_{w}=\left(1+\frac{4 A}{a / H}\right) \cos \theta
$$

where $A$ is a parameter which has the same meaning before. The CA of ODA-FLGs on smooth surface is $133.7^{\circ}$, so the predicted CA of ODA-FLGs on microstructure is $170.0^{\circ}$. But the real CA is smaller: $152.0^{\circ}$. According to reference [33], the main reason may be that the $35 \mu \mathrm{m}$ interval between the structures is too big, so some liquid can move into the interval. This phenomenon would reduce the CA and lower the surface's superhydrophobicity, and the real contact mode would not be the ideal Cassie mode, but would be between Cassie and Wenzel. If we can build a microstructure which has smaller intervals, the CA result may be closer to the predicted result. We will improve the experimental method, and try to finish the experiment using a smaller-interval wafer in the future.

In summary, we demonstrate the procedure of producing FLGs using MPCVD and the steps of chemical modification. Both hydrophilic and hydrophobic FLGs surfaces on smooth silicon substrate were prepared. SEM, HRTEM, EDX, XRD and Raman spectrum were used to characterize the properties of FLGs, O-FLGs and ODA-FLGs. Microstructures with different FLGs were prepared both superhydrophilic $\left(\mathrm{CA}=0^{\circ}\right)$ and superhydrophobic $(\mathrm{CA}=$ $152^{\circ}$ ) FLGs on the surface of silicon microstructures. These superhydrophilic/superhydrophobic surfaces have many important applications such as waterproof surfaces, anti-contaminant surfaces, coatings and biomedical devices.

\section{Methods}

Materials. Four-inch N-doped silicon wafers (111) were obtained from GRETEK Inc. Sulfuric acid (95 98\%) was purchased from Sinopharm Chemical Reagent Co., Beijing, China. Potassium permanganate $(99.5 \%)$ and sodium nitrate $(99 \%)$ was obtained from Beijing Chemical Reagent Co., Ltd., Beijing, China. Thionyl chloride (99\%) and dimethylformamide (DMF, 99\%) were obtained from Bodi Chemical Reagent Co., Tianjin, China. Octadecylamine (ODA, 90\%) was purchased from Aldrich Chemical Company Inc.

Preparation of the vertical few-layer graphene nanosheets. The FLGs were prepared in a microwave plasma chemical vapor deposition system. Silicon (111) was washed by acetone and then distilled water, for $10 \mathrm{~min}$ each. Silicon substrate was placed in a quartz boat and moved into the quartz tube. Then the reaction tube was pumped down to $5 \mathrm{~Pa}$, and $10 \mathrm{sccm} \mathrm{CH} 4$ and $50 \mathrm{sccm} \mathrm{H} 2$ were introduced into the reaction tube. After the pressure was adjusted to 7.5 Torr, a microwave was turned on to start the reaction and kept on for 135 seconds. The samples were cooled down to room temperature naturally under vacuum conditions. The black product was obtained on the surface of the substrate.

Chemical modification of vertical few-layer graphene nanosheets. In the first step of chemical modification, $23 \mathrm{ml}$ sulfuric acid and $0.5 \mathrm{~g}$ sodium nitrate were added into a $100 \mathrm{ml}$ flask, and $3 \mathrm{~g}$ potassium permanganate was stirred into the solution slowly at room temperature. This dark green solution was prepared for the oxidized FLGs. We put the silicon substrate upon which FLGs had been grown into the solution for 15 minutes, and then washed it by $3 \%$ peroxide and distilled water and dried it. In the second step $20 \mathrm{ml}$ thionyl chloride and $1 \mathrm{ml}$ dimethylformamide (DMF) were added into $50 \mathrm{ml}$ flask. After stirring, O-FLGs were put into the solution. After 24 hours of reaction, the silicon piece was washed by toluene and ethanol and dried. In the final step $10 \mathrm{~g}$ ODA was added into a $50 \mathrm{ml}$ flask, and was heated to $100^{\circ} \mathrm{C}$ in an oil bath. Finally the results were put into molten ODA for 4 hours. After the reaction, the silicon piece was cooled down and washed by a great deal of ethanol and dried.

Characterization of graphene nanosheets. Field-emission scanning electron microscopy (SEM, XL-SFEG, FEI Corp) was used for morphological observation of graphene sheets. Energy dispersive X-ray spectroscopy (EDX, XL-SFEG, FEI Corp) was employed to analyze components of samples. X-ray diffraction (XRD) was performed on an X'Pert Prodiffractometer with $\mathrm{Cu}$ Ka radiation at a wavelength of $\lambda=0.15418 \mathrm{~nm}$. Raman spectrum was recorded using JY-HR800 Raman microscope $(\lambda=532 \mathrm{~nm})$. The contact angles were measured by a POWERREACH contact angle measuring instrument. The default volume of droplets is $5 \mu \mathrm{l}$, though this is not mentioned explicitly in the article.

1. Nakajima, A., Hashimoto, K. \& Watanabe, T. Recent Studies on SuperHydrophobic Films. Monatsh. Chem. 132, 31-41 (2001).

2. Barthlott, W. \& Neinhuis, C. Purity of the sacred lotus, or escape from contamination in biological surfaces. Planta 202, 1-8 (1997).

3. Neinhuis, C. \& Barthlott, W. Characterization and Distribution of Waterrepellent, Self-cleaning Plant Surfaces. Ann. Bot 79, 667-677 (1997).

4. Geim, A. K. \& Novoselov, K. S. The rise of graphene. Nature Mater. 6, 183-191 (2007).

5. Peierls, R. E. Quelques proprieties typiques des corps solides. Ann. IHPoincare 5, 177-222 (1935).

6. Landau, L. D. Zur Theorie der Phasenumwandlungen. II. Phys Z. Sowjetunion 11, 26-35 (1937).

7. Novoselov, K. S. et al. Electric Field Effect in Atomically Thin Carbon Films. Science 306, 666-669 (2004).

8. Novoselov, K. S. et al. Two-dimensional atomic crystals. PNAS 102, 10451-10453 (2005).

9. Lee, C. G. et al. Measurement of the Elastic Properties and Intrinsic Strength of Monolayer Graphene. Science 321, 385-388 (2008).

10. Nair, R. R. et al. Fine Structure Constant Defines Visual Transparency of Graphene. Science 320, 1308 (2008).

11. Balandin, A. A. et al. Superior Thermal Conductivity of Single-Layer Graphene. Nano Lett. 8, 902-907 (2008).

12. Novoselov, K. S. et al. Two-dimensional gas of massless Dirac fermions in graphene. Nature 438, 197-200 (2005).

13. Berger, C. et al. Ultrathin Epitaxial Graphite: 2D Electron Gas Properties and a Route toward Graphene-based Nanoelectronics. . J. Phys Chem. B 108, 19912-19916 (2004).

14. Pan, Y. et al. Highly Ordered, Millimeter-Scale, Continuous, Single-Crystalline Graphene Monolayer Formed on Ru (0001). Adv. Mater 21, 2777-2780 (2009).

15. Li, X. S. et al. Large-Area Synthesis of High-Quality and Uniform Graphene Films on Copper Foils. Science 324, 1312-1314 (2009).

16. Stankovich, S. et al. Synthesis of graphene-based nanosheets via chemical reduction of exfoliated graphite oxide. Carbon 45, 1558-1565 (2007).

17. Rafiee, J., Rafiee, M. A., Yu, Z. Z. \& Koratkar, N. Superhydrophobic to Superhydrophilic Wetting Control in Graphene Films. Adv. Mater 22, 2151-2154 (2010).

18. Lin, Z., Liu, Y. \& Wong, C. Facile Fabrication of Superhydrophobic Octadecylamine-Functionalized Graphite Oxide Film. Langmuir 26, 16110-16114 (2010).

19. Zhang, L. et al. Formation of Superhydrophobic Microspheres of Poly(vinylidene fluoride-hexafluoropropylene)/Graphene Composite via Gelation. Langmuir 27, 8943-8949 (2011).

20. Zha, D. A. et al. Superhydrophobic polyvinylidene fluoride/graphene porous materials. Carbon 49, 5166-5172 (2011).

21. Huang, S. et al. The preparation of graphene hybrid films decorated with poly [2-methoxy-5-(2'-ethyl-hexyloxy)-1,4-phenylene vinylene] particles prepared by non-solvent induced precipitation. Carbon 50, 216-224 (2012).

22. Lin, Y. R., Ehlert, G. J., Bukowsky, C. \& Sodano, H. A. Superhydrophobic Functionalized Graphene Aerogels. Applied Materials \& Interfaces 3, 2200-2203 (2011).

23. Malesevic, A. et al. Field emission from vertically aligned few-layer graphene. J. Appl Phys. 104, 084301 (2008).

24. Zhu, M. Y. et al. A mechanism for carbon nanosheet formation. Carbon $\mathbf{4 5}$, 2229-2234 (2007). 
25. Oner, D. \& McCarthy, T. J. Ultrahydrophobic Surfaces. Effects of Topography Length Scales on Wettability. Langmuir 16, 7777-7782 (2000).

26. Dresselhaus, M. S., Jorio, A., Hofmann, M., Dresselhaus, G. \& Saito, R. Perspectives on Carbon Nanotubes and Graphene Raman Spectroscopy. Nano Lett 10, 751-758 (2010).

27. Malard, L. M., Pimenta, M. A., Dresselhaus, G. \& Dresselhaus, M. S. Raman spectroscopy in graphene. Physics Reports 473, 51-87 (2009).

28. Tang, B., Guoxin, H. \& Gao, H. Raman Spectroscopic Characterization of Graphene. Applied Spectroscopy Reviews 45, 369-407 (2010).

29. Casiraghi, C., Pisana, S., Novoselov, K. S., Geim, A. K. \& Ferrari, A. C. Raman fingerprint of charged impurities in graphene. Applied Physics Letters 91, 233108 (2007).

30. Ferrari, A. C. et al. Raman Spectrum of Graphene and Graphene Layers. Physical Review Letters 97, 187401 (2006).

31. Wenzel, R. N. Resistance of solid surfaces to wetting by water. Ind. Eng. Chem. 28, 988 (1936).

32. Cassie, A. B. D. \& Baxter, S. Wettability of porous surfaces. Trans. Faraday Soc. 40, 546-551 (1944).

33. Patankar, A. N. Transition between Superhydrophobic States on Rough Surfaces. Langmuir, 20, 7097-7102 (2004).

\section{Acknowledgements}

The research presented in this paper was financially supported by the National Key Basic Research and Development Program of China (the 973 Program) through grant No. 2011 CB932703.

\section{Author contributions}

J.D., T.Z.Y. and L.L.J. carried out the experiments under the guidance of Z.H.Y. and C.M.S.; J.D. performed the theoretical calculations; Z.H.Y. coordinated the project; J.D. analyzed the data; J.D., Z.H.Y. and C.M.S. wrote the paper. All authors discussed the results and commented on the manuscript.

\section{Additional information}

Competing financial interests: The authors declare no competing financial interests.

License: This work is licensed under a Creative Commons

Attribution-NonCommercial-NoDerivs 3.0 Unported License. To view a copy of this license, visit http://creativecommons.org/licenses/by-nc-nd/3.0/

How to cite this article: Dong, J., Yao, Z.H., Yang, T.Z., Jiang, L.L. \& Shen, C.M. Control of Superhydrophilic and Superhydrophobic Graphene Interface. Sci. Rep. 3, 1733; DOI:10.1038/srep01733 (2013). 